\title{
Decomposability of graphs into subgraphs fulfilling the 1-2-3 Conjecture
}

\author{
Julien Bensmail ${ }^{\mathrm{a}, 1}$, Jakub Przybyło ${ }^{\mathrm{b}, 2}$ \\ ${ }^{a}$ Université Côte d'Azur, CNRS, I3S, Inria, France \\ ${ }^{b}$ AGH University of Science and Technology, Faculty of Applied Mathematics, al. A. Mickiewicza 30, \\ 30-059 Krakow, Poland
}

\begin{abstract}
The well-known 1-2-3 Conjecture asserts that the edges of every graph without isolated edges can be weighted with 1,2 and 3 so that adjacent vertices receive distinct weighted degrees. This is open in general. We prove that every $d$-regular graph, $d \geq 2$, can be decomposed into at most 2 subgraphs (without isolated edges) fulfilling the 1-23 Conjecture if $d \notin\{10,11,12,13,15,17\}$, and into at most 3 such subgraphs in the remaining cases. Additionally, we prove that in general every graph without isolated edges can be decomposed into at most 24 subgraphs fulfilling the 1-2-3 Conjecture, improving the previously best upper bound of 40 . Both results are partly based on applications of the Lovász Local Lemma.
\end{abstract}

Keywords: 1-2-3 Conjecture, locally irregular graph, graph decomposition

\section{Introduction}

A graph of order at least 2 cannot be irregular, i.e. its vertices cannot have pairwise distinct degrees. This does not concern multigraphs though. The least $k$ so that an irregular multigraph can be obtained from a given graph $G$ by replacing each edge by at most $k$ parallel edges is called the irregularity strength of $G$. This graph invariant was introduced in [11], and investigated further in numerous papers as a particular mean for measuring the "level of irregularity" of graphs, see e.g. [3, 12, 13, 14, 15, 18, 21, 23, 25, 26, 27]. Potential alternative definitions of "irregular graphs" were also investigated by Chartrand, Erdös and Oellermann in [10]. In [5] the authors introduced and initiated research devoted to so-called locally irregular graphs, i.e. graphs in which adjacent vertices have distinct degrees. Already earlier a local version of irregularity strength was studied in 20]. There Karoński, Łuczak and Thomason considered the least $k$ so that a locally irregular multigraph can be obtained from a given graph $G=(V, E)$ via, again, replacement of every edge with at most $k$ parallel edges. This problem was in fact originally formulated in terms of weightings, where by a $k$-edge-weighting of $G$

\footnotetext{
${ }^{1}$ The first author was supported by PEPS grant POCODIS.

${ }^{2}$ This work was partially supported by the Faculty of Applied Mathematics AGH UST statutory tasks within subsidy of Ministry of Science and Higher Education.

Preprint submitted to Elsevier

November 9, 2018
} 
we mean any mapping $\omega: E \rightarrow\{1,2, \ldots, k\}$. For such $w$ we may define the so-called weighted degree of or simply the sum at a given vertex $v$ as:

$$
s_{\omega}(v):=\sum_{e \in E_{v}} \omega(e),
$$

where $E_{v}$ denotes the set of edges incident with $v$ in $G$; we shall usually write simply $s(v)$ instead of $s_{\omega}(v)$ if this causes no ambiguities further on. So in this setting, the authors of [20] were interested in the least $k$ such that a $k$-edge-weighting $\omega$ of $G$ exists so that $s_{\omega}(u) \neq s_{\omega}(v)$ for every edge $u v \in E$ - we say that $u$ and $v$ are sum-distinguished then (note we must assume that $G$ contains no isolated edges to that end, i.e. that it has no $K_{2}$-components). They posed a very intriguing question, commonly known as the 1-2-3 Conjecture in the literature nowadays.

Conjecture 1 (1-2-3 Conjecture). For every graph $G=(V, E)$ without isolated edges there exists a weighting $\omega: E \rightarrow\{1,2,3\}$ sum-distinguishing all neighbours in $G$.

They confirmed it for 3-colourable graphs, i.e. for graphs with $\chi(G) \leq 3$.

Theorem 2 ([20]). Every 3-colourable graph without isolated edges fulfills the 1-2-3 Conjecture.

This is also commonly known to hold in particular for complete graphs. In general the conjecture is however still widely open. The first constant upper bound, with 30 instead of 3, was provided in [1] , and then improved in [2] and [31]. The best general result thus far was delivered by Kalkowski, Karoński and Pfender, who proved that it is sufficient to use weights $1,2,3,4,5$, see [19]. This result was obtained via refinement and modification of an algorithm developed by Kalkowski 17] (concerning a total analogue of the 1-2-3 Conjecture, see e.g. [29]). Quite recently a complete characterization of bipartite graphs for which it is sufficient to use just weights 1 and 2 was also provided by Thomassen, Wu and Zhang [30]. Note that graphs which require only one weight (i.e. 1) are precisely the locally irregular graphs.

Another direction of research towards inducing local irregularity in a graph was developed by Baudon et al. [5], this time via graph decompositions. In this paper, by a decomposition of a graph $G$ we mean a partition of the set of its edges into subsets inducing subgraphs of $G$ (usually with some specified features). We say a graph $G=(V, E)$ can be decomposed into $k$ locally irregular subgraphs if $E$ can be partitioned into $k$ sets: $E=E_{1} \cup E_{2} \cup \ldots \cup E_{k}$ so that $G_{i}:=\left(V, E_{i}\right)$ is locally irregular (where we admit $E_{i}$ to be empty) for $i=1,2, \ldots, k$. Equivalently, it means we may colour the edges of $G$ with at most $k$ colours so that each of these induces a locally irregular subgraph in $G$. In $[5]$ it was conjectured that except for some family of exceptional graphs (each of which has maximum degree at most 3, see [5] for details), every connected graph can be decomposed into 3 locally irregular subgraphs. This was then confirmed in [28] for graphs with sufficiently large minimum degree.

Theorem $3([\mathbf{2 8}])$. Every graph $G$ with minimum degree $\delta(G) \geq 10^{10}$ can be decomposed into 3 locally irregular subgraphs. 
In general it was also proved by Bensmail, Merker and Thomassen [9] that every connected graph which is not exceptional can be decomposed into (at most) 328 locally irregular subgraphs, what was then pushed down to 220 such subgraphs by Lužar, Przybyło and Soták [22]. See also [5, 6, 9, 22] for a number of partial and related results.

Here we develop research initiated in 7], and related to the both concepts discussed above. From now on we shall write a graph fufills the 1-2-3 Conjecture if there actually exists its neighbour sum-distinguishing 3-edge-weighting (assuming this holds in particular for an edgeless graph). Though we are not yet able to prove the 1-2-3 Conjecture, even in the case of regular graphs, we shall prove below that for almost every $d \geq 2$, a $d$-regular graph $G$ can be decomposed into 2 subgraphs fulfilling the 1-2-3 Conjecture, while in the remaining cases it can be decomposed into 3 such subgraphs. At the end we shall additionally prove that in general every graph without isolated edges can be decomposed into (at most) 24 subgraphs consistent with the 1-2-3 Conjecture, while thus far it was known that 40 such subgraphs were always sufficient, see [7] (also for other related results).

\section{Basic Tools}

We first present one basic observation followed by a recollection of a few fundamental tools of the probabilistic method we shall use later on. For a vertex $v$ of a given graph $G=(V, E)$, by $d_{S}(v)$ we mean the number of edges $u v \in E$ with $u \in S$ if $S \subseteq V$, or the number of edges $u v \in S$ in the case when $S \subseteq E$.

Observation 4. Every bipartite graph $G$ can be decomposed into two subgraphs $G_{1}$ and $G_{2}$ such that for every vertex $v$ of $G$,

$$
d_{G_{1}}(v) \in\left[\frac{d_{G}(v)-1}{2}, \frac{d_{G}(v)+1}{2}\right] .
$$

Proof. If the set $U$ of the vertices of odd degree in $G$ is nonempty, then add a new vertex $u$ and join it by a single edge with every vertex in $U$; denote the obtained graph by $G^{\prime}$. If $U=\emptyset$, set $G^{\prime}=G$ and denote any vertex of $G^{\prime}$ as $u$. As the degrees of all vertices in $G^{\prime}$ are even, there exists an Eulerian tour in it. We then start at the vertex $u$ and traverse all edges of $G^{\prime}$ once along this Eulerian tour colouring them alternately red and blue. Then the red edges in $G$ induce its subgraph $G_{1}$ consistent with our requirements. This follows from the fact that if all degrees in the bipartite graph $G$ are even, then it has to have an even number of edges, and thus the thesis holds in particular for $u$.

The following standard versions of the Lovász Local Lemma can be found e.g. in [4].

Theorem 5 (The Local Lemma; Symmetric Version). Let $\mathcal{A}$ be a finite family of events in any probability space. Suppose that every event $A \in \mathcal{A}$ is mutually independent of a set of all the other events in $\mathcal{A}$ but at most $D$, and that $\operatorname{Pr}(A) \leq p$ for each $A \in \mathcal{A}$. If

$$
e p(D+1) \leq 1,
$$

then $\operatorname{Pr}\left(\bigcap_{A \in \mathcal{A}} \bar{A}\right)>0$. 
Theorem 6 (The Local Lemma; General Case). Let $\mathcal{A}$ be a finite family of events in any probability space and let $D=(\mathcal{A}, E)$ be a directed graph such that every event $A \in \mathcal{A}$ is mutually independent of all the events $\{B:(A, B) \notin E\}$. Suppose that there are real numbers $x_{A}(A \in \mathcal{A})$ such that for every $A \in \mathcal{A}, 0 \leq x_{A}<1$ and

$$
\operatorname{Pr}(A) \leq x_{A} \prod_{B \leftarrow A}\left(1-x_{B}\right) .
$$

Then $\operatorname{Pr}\left(\bigcap_{A \in \mathcal{A}} \bar{A}\right)>0$.

Here $B \leftarrow A$ (or $A \rightarrow B$ ) means that there is an arc from $A$ to $B$ in $D$, the so-called dependency digraph. The Chernoff Bound below can be found e.g. in [16] (Th. 2.1, page $26)$.

Theorem 7 (Chernoff Bound). For any $0 \leq t \leq n p$,

$$
\operatorname{Pr}(\operatorname{BIN}(n, p)>n p+t)<e^{-\frac{t^{2}}{3 n p}} \quad \text { and } \quad \operatorname{Pr}(\operatorname{BIN}(n, p)<n p-t)<e^{-\frac{t^{2}}{2 n p}}
$$

where $\operatorname{BIN}(n, p)$ is the sum of $n$ independent Bernoulli variables, each equal to 1 with probability $p$ and 0 otherwise.

\section{Main Result for Regular Graphs}

In this section we shall prove that for almost all integers $d \geq 2$, every $d$-regular graph can be decomposed into two graphs fulfilling the 1-2-3 Conjecture, while in the remaining few cases - into three such graphs. The first subsection below is devoted to small values of $d$; more generally we investigate in it graphs with upper-bounded chromatic number.

\subsection{Graphs with Bounded Chromatic Number}

In [7] it was proved the following result (note it follows also by Corollary 11] below).

Theorem $8([7])$. Every graph $G$ without isolated edges and with $\chi(G) \leq 9$ can be decomposed into 2 graphs fulfilling the 1-2-3 Conjecture.

As complete graphs are known to fulfill the 1-2-3 Conjecture, by Brooks' Theorem we thus obtain that every $d$-regular graph with $2 \leq d \leq 9$ can be decomposed into 2 graphs fulfilling the 1-2-3 Conjecture. In order to first achieve the main result of this paper for the case of regular graphs with upper-bounded degree, we shall generalize Theorem 8 , For this aim we use the following Lemma 9, which is very similar to a one proved in [7]. We however present an alternative brief proof of this slightly modified version for the sake of completeness of the exposition of our reasoning.

Lemma 9. If the edges of a graph $G$ without isolated edges can be 2-coloured with red and blue so that the induced red subgraph $R$ and blue subgraph $B$ satisfy $\chi(R) \leq r \geq 3$ and $\chi(B) \leq b \geq 3$, then we can also do it in such a way that neither $B$ nor $R$ contains an isolated edge. 
PRoOF. Start from a 2-colouring of the edges of $G$ with $\chi(R) \leq r \geq 3$ and $\chi(B) \leq b \geq 3$ which minimizes the number of monochromatic $K_{2}$-components (note that each isolated triangle of $G$ shall be monochromatic then). We shall show that if the number of these is still positive, then we may "get rid" of any given such component, without creating a new one (thus getting a contradiction, and hence proving the thesis):

Assume $u v$ forms such a monochromatic, say blue $K_{2}$-component. Observe that:

(1') $u$ and $v$ must belong to the same component of $R$, as otherwise one may recolour $u v$ red;

(2') if $e$ is a red edge adjacent with $u v$, say $e=u w$, then the size $k$ of the (red) component of $R-e$ including $w$ equals 1 (hence, all red paths originating at $u$ or $v$ must be of length 2 , i.e. there are in particular no isolated red edges adjacent with $u v$ ), as otherwise, if there was any such edge $e$ with $k=0$, we could recolour $u w$ blue (we would not create any red $K_{2}$-component then, as we would not change colours on some existing red path joining $u$ and $v$ ), while in the remaining cases (i.e. when $k \geq 2$ for each red edge $e$ adjacent with $u v$ ) we could also recolour $u w$ blue.

By $\left(1^{\prime}\right)$ and (2') there must be a red path $u w v$ in $G$, and hence, by $\left(2^{\prime}\right), d(u)=2=$ $d(v)$. Therefore, as all isolated triangles of $G$ are monochromatic, $d(w) \geq 3$, and thus by (2') all edges incident with $w$ except for $u w$ and $v w$ are blue. We may however recolour $u v$ red and $u w$ blue then.

Lemma 10. For each positive integer $k$, every graph $G=(V, E)$ without isolated edges and with $\chi(G) \leq 3^{k}$ can be decomposed into $k$ (some possibly empty) subgraphs $G_{1}, G_{2}$, $\ldots, G_{k}$ such that $\chi\left(G_{i}\right) \leq 3$ and $G_{i}$ contains no isolated edges for $i=1,2, \ldots, k$.

ProOF. We prove the lemma by induction with respect to $k$. For $k=1$ it trivially holds, so let us assume that $k \geq 2$. Partition $V$ into (possibly empty) independent sets $V_{1}, V_{2}, \ldots, V_{3^{k}}$. Colour red every edge $u v \in E$ such that $u \in V_{i}$ and $v \in V_{j}$ with $i \equiv j(\bmod 3)$, and colour blue the remaining edges of $G$. Let $R$ and $B$ be the red and, resp., blue subgraphs of $G$. Note that $\chi(B) \leq 3$, as $V_{1+r} \cup V_{4+r} \cup V_{7+r} \cup \ldots \cup V_{3^{k}-2+r}$ forms an independent set in $B$ for $r=0,1,2$. On the other hand, $\chi(R) \leq 3^{k-1}$, as there are no edges in $R$ between any two of the three red subgraphs $G\left[V_{1+r} \cup V_{4+r} \cup V_{7+r} \cup \ldots \cup V_{3^{k}-2+r}\right]$ with $r=0,1,2$. Note that by Lemma 9, we may assume that neither $B$ nor $R$ contains an isolated edge. By the induction hypothesis, $R$ can be then decomposed into subgraphs $G_{1}, G_{2}, \ldots, G_{k-1}$ with $\chi\left(G_{i}\right) \leq 3$ none of which contains an isolated edge. Setting $G_{k}:=B$ thus yields the thesis.

By Lemma 10 and Theorem 2 we obtain the following corollaries.

Corollary 11. Every graph $G$ without isolated edges can be decomposed into $\left\lceil\log _{3} \chi(G)\right\rceil$ graphs fulfilling the 1-2-3 Conjecture.

Corollary 12. Every d-regular graph $G$ with $10 \leq d \leq 17$ can be decomposed into 3 subgraphs fulfilling the 1-2-3 Conjecture.

To prove divisibility into 2 such subgraphs of $d$-regular graphs with larger $d$, we first prove in the next Subsection 3.2 that they admit a special vertex partition. In Subsection 3.3. we then discuss a peculiar sufficient condition for a graph to fulfill the 1-2-3 Conjecture. 


\subsection{Random Vertex Partition}

Lemma 13. The vertices of every d-regular graph $G$ with $d \geq 14, d \neq 15,17$, can be partitioned into sets $V_{0}$ and $V_{1}$ such that if $d \equiv r \bmod 2$ for some $r \in\{0,1\}$, then:

(i) $\forall v \in V_{0}: d_{V_{0}}(v) \geq 2+r$;

(ii) $\forall v \in V_{0}: d_{V_{1}}(v) \geq 2$;

(iii) $\forall v \in V_{1}: d_{V_{1}}(v) \geq 2+r$;

(iv) $\forall v \in V_{1}: d_{V_{0}}(v) \geq 2$.

Proof. Assume $G=(V, E)$ is a $d$-regular graph with $d \geq 14, d \neq 15,17$. To every vertex we randomly and independently assign 0 or 1 - each with probability $1 / 2$, and denote the 2-colouring obtained by $c$. Set $V_{0}=c^{-1}(0), V_{1}=c^{-1}(1)$.

Assume first that $d$ is even (i.e., $r=0$ ). For any given vertex $v \in V$, denote by:

- $A_{1}(v)$ - the event that $v \in V_{0}$ and $d_{V_{0}}(v) \leq 1$;

- $A_{2}(v)$ - the event that $v \in V_{0}$ and $d_{V_{1}}(v) \leq 1$;

- $A_{3}(v)$ - the event that $v \in V_{1}$ and $d_{V_{1}}(v) \leq 1$;

- $A_{4}(v)$ - the event that $v \in V_{1}$ and $d_{V_{0}}(v) \leq 1$.

Note that if we prove that none of these events holds for some (random) colouring $c$, then the thesis shall be fulfilled. As drawings for all vertices are independent, for every $v \in V$ we have:

$$
\begin{aligned}
\mathbf{P} r\left(A_{1}(v)\right) & =\mathbf{P} r\left(v \in V_{0}\right) \cdot \mathbf{P} r\left(d_{V_{0}}(v) \leq 1\right) \\
& =\mathbf{P} r\left(v \in V_{0}\right) \cdot\left[\mathbf{P} r\left(d_{V_{0}}(v)=0\right)+\mathbf{P} r\left(d_{V_{0}}(v)=1\right)\right] \\
& =\frac{1}{2} \cdot\left[\left(\frac{1}{2}\right)^{d}+d\left(\frac{1}{2}\right)^{d}\right]=(1+d)\left(\frac{1}{2}\right)^{d+1},
\end{aligned}
$$

and analogously, for every $i=2,3,4$,

$$
\mathbf{P} r\left(A_{i}(v)\right)=(1+d)\left(\frac{1}{2}\right)^{d+1} .
$$

Note now that every event $A_{i}(v)$ is mutually independent of all other events $A_{j}(u)$ with $u$ at distance at least 3 from $v$, i.e. of all but at most $4 d^{2}+3$ other events. Therefore, by (1), (3) and (4), in order to apply the Lovász Local Lemma it is sufficient to show that:

$$
e(1+d)\left(\frac{1}{2}\right)^{d+1}\left(4 d^{2}+4\right)<1
$$

for every even integer $d \geq 14$. For $d=14$ the left-hand side of inequality (5) takes value (approximately) $0.9805 \ldots<1$, while for $d \geq 16$ inequality (5) is implied by the following one:

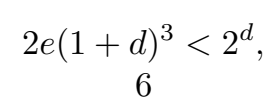


equivalent to:

$$
\sqrt[3]{2 e}(1+d)<2^{\frac{d}{3}}
$$

which holds as for $f(d):=2^{d / 3}-\sqrt[3]{2 e}(1+d)$, we have $f^{\prime}(d)=2^{d / 3} 1 / 3 \ln 2-\sqrt[3]{2 e}>0$ for $d \geq 16$ and $f(16) \approx 10.4253>0$.

By (3), (4), (5) and Theorem 5 we thus conclude that

$$
\mathbf{P} r\left(\bigcap_{v \in V}\left(\overline{A_{1}(v)} \cap \overline{A_{2}(v)} \cap \overline{A_{3}(v)} \cap \overline{A_{4}(v)}\right)\right)>0 .
$$

The thesis follows.

Assume now that $d$ is odd (i.e., $r=1$ ) and $d \geq 19$. In order to optimize our approach we shall now have to aggregate the events concerning our requirements (i)-(iv). Thus for a vertex $v \in V$, denote the following (aggregated) event:

- $B(v):\left(v \in V_{0} \wedge d_{V_{0}}(v) \geq 3 \wedge d_{V_{1}}(v) \geq 2\right) \vee\left(v \in V_{1} \wedge d_{V_{1}}(v) \geq 3 \wedge d_{V_{0}}(v) \geq 2\right)$.

In order to prove the thesis it is then enough to apply the Local Lemma to show that the probability that $B(v)$ holds for every $v \in V$ is positive.

Note that for every $v \in V$,

$$
\begin{aligned}
\mathbf{P} r(\overline{B(v)})= & \mathbf{P} r\left(\left(v \in V_{1} \vee d_{V_{0}}(v) \leq 2 \vee d_{V_{1}}(v) \leq 1\right) \wedge\left(v \in V_{0} \vee d_{V_{1}}(v) \leq 2 \vee d_{V_{0}}(v) \leq 1\right)\right) \\
= & \frac{1}{2} \mathbf{P} r\left(\overline{B(v)} \mid v \in V_{1}\right)+\frac{1}{2} \mathbf{P} r\left(\overline{B(v)} \mid v \in V_{0}\right) \\
= & \mathbf{P} r\left(\overline{B(v)} \mid v \in V_{1}\right) \\
= & \mathbf{P} r\left(d_{V_{1}}(v) \leq 2 \vee d_{V_{0}}(v) \leq 1\right) \\
= & \mathbf{P} r\left(d_{V_{1}}(v)=2\right)+\mathbf{P} r\left(d_{V_{1}}(v)=1\right)+\mathbf{P} r\left(d_{V_{1}}(v)=0\right) \\
& +\mathbf{P} r\left(d_{V_{0}}(v)=1\right)+\mathbf{P} r\left(d_{V_{0}}(v)=0\right) \\
= & \left(\begin{array}{l}
d \\
2
\end{array}\right)\left(\frac{1}{2}\right)^{d}+d\left(\frac{1}{2}\right)^{d}+\left(\frac{1}{2}\right)^{d}+d\left(\frac{1}{2}\right)^{d}+\left(\frac{1}{2}\right)^{d} \\
= & \left(\frac{d(d-1)}{2}+2 d+2\right)\left(\frac{1}{2}\right)^{d} \\
< & (d+2)^{2} 2^{-(d+1)} .
\end{aligned}
$$

Again an event $\overline{B(v)}$ is mutually independent of all other events $\overline{B(u)}$ with $u$ at distance at least 3 from $v$, i.e. of all but at most $d^{2}<(d+2)^{2}-1$ other events. Moreover, the following inequality:

$$
e(d+2)^{2} 2^{-(d+1)}(d+2)^{2}<1
$$

is fulfilled for every $d \geq 19$, as this is equivalent to the fact that $g(d):=2^{(d+1) / 4}-$ $\sqrt[4]{e}(d+2)>0$ (for $d \geq 19)$. This in turn holds, since $g(19) \approx 5.0355>0$ and $g^{\prime}(d)=$ $2^{(d-7) / 4} \ln 2-\sqrt[4]{e}>0$ for $d \geq 19$.

By (6), (7) and Theorem [5 we thus obtain that

$$
\mathbf{P} r\left(\bigcap_{v \in V} B(v)\right)>0 .
$$




\subsection{Family of Graphs Fulfilling the 1-2-3 Conjecture}

For a subset $S$ of the set of vertices of a given graph $G, G[S]$ shall denote the subgraph induced by $S$ in $G$, while by $G_{1} \cup G_{2}$ we shall mean the sum of two graphs $G_{1}=\left(V_{1}, E_{1}\right)$, $G_{2}=\left(V_{2}, E_{2}\right)$ understood as the pair $\left(V_{1} \cup V_{2}, E_{1} \cup E_{2}\right)$. An independent set in a graph $G=(V, E)$ is a subset $I$ of $V$ such that no edge of $G$ has both ends in $I$. We call it maximal (or an independent dominating set) if every vertex in $V \backslash I$ has a neighbour in $I$. In order to prove the existence of a specific family of graphs fulfilling the 1-2-3 Conjecture we shall apply in the following lemma a certain refinement of Kalkowki's algorithm from [17], exploiting for this aim the concept of maximal independent sets; see [8] for a corresponding application of a mixture of these two ingredients.

Lemma 14. If a graph $G=(V, E)$ contains a maximal independent set $I$ such that there exists a constant $\alpha \geq 1$ so that for $R:=V \backslash I$,

$\left(1^{\circ}\right) d(v) \leq \alpha$ for every $v \in I$ and

$\left(2^{\circ}\right) d(v) \geq \alpha+\frac{d_{R}(v)+1}{2}$ for every $v \in R$,

then $G$ fulfills the 1-2-3 Conjecture.

Proof. By $\left(2^{\circ}\right), d(v) \geq 2$ for every $v \in R$, and thus there are no isolated edges in $G$. Note also that since $I$ is a maximal independent set in $G$, then:

$\left(3^{\circ}\right) d_{I}(v) \geq 1$ for every $v \in R$,

so for every vertex $v \in R$ we may fix an edge $e_{v}$ joining $v$ with some vertex in $I$.

We shall construct a 3 -edge-weighting of $G$ sum-distinguishing its neighbours. Initially we label all edges in $G$ by 2 . These shall be modified gradually, and by $\omega(e)$ we shall always understand the current weight of an edge $e$ in a given moment of our ongoing relabelling algorithm specified below, and similarly, by $s(v)$ we shall understand the current sum at a vertex $v$ in $G$.

Let $G_{R}=G[R]$ be the graph induced by $R$ in $G$. We analyse every of its components one by one, in any fixed order, and modify the labels of some of the edges incident (in $G$ ) with at least one vertex of this component. Suppose $H$ is the next component to be analysed within the on-going algorithm, and arbitrarily order its vertices linearly into a sequence $v_{1}, v_{2}, \ldots, v_{n}$. We shall analyse one vertex in the sequence after another starting from $v_{1}$, for which we perform no changes. Suppose thus we are about to analyse a vertex $v_{j}$ with $j \geq 2$ (if $H$ has more than one vertex) and denote by $N_{H}^{-}\left(v_{j}\right)$ the set of neighbours of $v_{j}$ in $H$ which precede it in the fixed linear ordering - we call these the backward neighbours of $v_{j}$. Similarly we define the set $E_{H}^{-}\left(v_{j}\right)$ of the backward edges of $v_{j}$, i.e. these joining $v_{j}$ with its backward neighbours in $H$. We shall now modify (if necessary) weights of some edges, in order to obtain a sum at $v_{j}$ (in $G$ ) which is distinct from the sums of all its backward neighbours - this sum of $v$ shall then not change in the further part of the algorithm. To obtain our goal we shall be allowed to perform changes only on the edges incident with its backward neighbours, namely for every backward edge $v_{k} v_{j} \in E_{H}^{-}\left(v_{j}\right)$ of $v_{j}$ (i.e. with $k<j$ ) we shall be allowed to modify the labels of $v_{k} v_{j}$ and $e_{v_{k}}$ so that the sum at $v_{k}$ does not change; more specifically, if prior to this step $j$ we had $\omega\left(e_{v_{k}}\right)=2$ (and $\omega\left(v_{k} v_{j}\right)=2$ ), then we may increase the label of $v_{k} v_{j}$ by 1 and decrease the label of $e_{v_{k}}$ by 1 (or perform no changes on these two edges), while 
if priory we had $\omega\left(e_{v_{k}}\right)=1$, we may decrease the label of $v_{k} v_{j}$ by 1 and increase the label of $e_{v_{k}}$ by 1 (observe that then $\omega\left(e_{v_{k}}\right) \in\{1,2\}$ and $\omega\left(v_{k} v_{j}\right) \in\{1,2,3\}$ ). Note that such admitted operations allow us to change the label of every backward edge of $v_{j}$ by exactly 1 (or do nothing with this label). Hence we have available at least $\left|E_{H}^{-}\left(v_{j}\right)\right|+1$ distinct sums at $v_{j}$ via these operations. We choose one of these sums which is distinct from the current sums of all backward neighbours of $v_{j}$ and denote it by $s^{*}$ (it exists, as $\left|E_{H}^{-}\left(v_{j}\right)\right|+1=\left|N_{H}^{-}\left(v_{j}\right)\right|+1$ ), and we perform (some of the) admissible changes described above so that $s\left(v_{j}\right)=s^{*}$ afterwards. As these changes do not influence sums (in $G$ ) of the other vertices of $H, v_{j}$ is now sum-distinguished from all its backward neighbours, and

$$
\begin{aligned}
s\left(v_{j}\right) & \geq 2 \cdot d_{I}\left(v_{j}\right)+1 \cdot d_{R}\left(v_{j}\right)=2\left(d\left(v_{j}\right)-d_{R}\left(v_{j}\right)\right)+d_{R}\left(v_{j}\right) \\
& =2\left(d\left(v_{j}\right)-\frac{d_{R}\left(v_{j}\right)}{2}\right) \geq 2 \alpha+1
\end{aligned}
$$

by $\left(2^{\circ}\right)$. This shall not change as we guarantee that the sums of $v_{1}, v_{2}, \ldots, v_{j}$ shall not be modified in the further part of the construction. After step $n$, all neighbours in $H$ are thus sum-distinguished (in $G$ ), and we continue in the same manner with a consecutive component of $G_{R}$, if any is still left. Note that performing such changes concerning one component of $G_{R}$ does not influence the sums in the other components, hence at the end of our construction all neighbours in $G_{R}$ are sum-distinguished (in $G$ ). On the other hand, as due to our algorithm every edge incident with a vertex in $I$ has final weight 1 or 2 , by $\left(1^{\circ}\right)$ we obtain that for $v \in I$,

$$
s(v) \leq 2 d(v) \leq 2 \alpha
$$

Hence, by (8) and (9), every vertex in $R$ is also sum-distinguished from each of its neighbours in $I$. As $I$ is an independent set, we thus obtain a desired 3-edge-weighting of $G$.

\subsection{Main Result for Almost All Degrees}

Theorem 15. Every $d$-regular graph $G$ with $d \geq 14, d \neq 15,17$, can be decomposed into two graphs fulfilling the 1-2-3 Conjecture.

Proof. Let $G=(V, E)$ be a $d$-regular graph with $d \geq 14, d \neq 15,17$, and $d \equiv r \bmod 2$ for some $r \in\{0,1\}$. Let then $V=V_{0} \cup V_{1}$ be a vertex partition consistent with the thesis of Lemma 13. Denote $G_{0}:=G\left[V_{0}\right], G_{1}:=G\left[V_{1}\right]$.

Let $H$ be the bipartite graph induced by the edges between $V_{0}$ and $V_{1}$. Then $\delta(H) \geq 2$ by (ii) and (iv) from Lemma [13. By Observation 4 we next decompose $H$ into two subgraphs $H_{0}$ and $H_{1}$ such that

$$
d_{H_{0}}(v) \in\left[\frac{d_{H}(v)-1}{2}, \frac{d_{H}(v)+1}{2}\right],
$$

and thus also

$$
d_{H_{1}}(v) \in\left[\frac{d_{H}(v)-1}{2}, \frac{d_{H}(v)+1}{2}\right]
$$

for every vertex $v \in V$. 
Let $G_{0}^{\prime}=G_{0} \cup H_{0}, G_{1}^{\prime}=G_{1} \cup H_{1}$. Obviously $G_{0}^{\prime}$ and $G_{1}^{\prime}$ constitute a decomposition of $G$. In order to finish the proof it is thus sufficient to prove that they are both consistent with the assumptions of Lemma 14. We show this to hold for $G_{0}^{\prime}$, as the reasoning for $G_{1}^{\prime}$ is precisely symmetrical.

For this aim note first that by the definition of $H_{0}$, the set $I:=V_{1}$ is an independent set in $G_{0}^{\prime}$, and by (ii) from Lemma 13 and (10) above, it is also maximal. We shall now show that $\left(1^{\circ}\right)$ and $\left(2^{\circ}\right)$ from Lemma 14 hold for $G_{0}^{\prime}$ with $R=V_{0}$ and $\alpha:=\frac{d-2-r}{2}$.

By (iii) from Lemma 13, for every vertex $v \in I=V_{1}$,

$$
d_{H}(v) \leq d-2-r,
$$

and hence, by (10):

$$
d_{G_{0}^{\prime}}(v)=d_{H_{0}}(v) \leq\left\lceil\frac{d_{H}(v)}{2}\right\rceil \leq\left\lceil\frac{d-2-r}{2}\right\rceil=\frac{d-2-r}{2}
$$

according to the definition of $r$. Consequently, $\left(1^{\circ}\right)$ holds.

On the other hand, by (10), for every $v \in R=V_{0}$ :

$$
\begin{aligned}
d_{G_{0}^{\prime}}(v) & =d_{G_{0}}(v)+d_{H_{0}}(v) \geq d_{V_{0}}(v)+\left\lfloor\frac{d-d_{V_{0}}(v)}{2}\right\rfloor \geq d_{V_{0}}(v)+\frac{d-d_{V_{0}}(v)-1}{2} \\
& =\frac{d+d_{V_{0}}(v)-1}{2} \geq \frac{d+d_{V_{0}}(v)-1-r}{2}=\frac{d-2-r}{2}+\frac{d_{V_{0}}(v)+1}{2} \\
& =\alpha+\frac{d_{R}(v)+1}{2},
\end{aligned}
$$

and thus $\left(2^{\circ}\right)$ holds.

\section{General Upper Bound for All Graphs}

We conclude by showing that every graph without isolated edges can be decomposed into a certain number $K$ of graphs fulfilling the 1-2-3 Conjecture, where $K \leq 24$. We thereby improve the previously best upper bound $K \leq 40$ from [7]. We start from proving a lemma on the existence of a subset of edges with certain properties in graphs with sufficiently large minimum degree.

Lemma 16. If $G=(V, E)$ is a graph with minimum degree $\delta \geq 10^{10}+10^{8}$, then there is a subset $S \subseteq E$ such that $1 \leq d_{S}(v) \leq d(v)-10^{10}$ for every vertex $v \in V$.

Proof. Let $\Delta=\Delta(G)$. For every vertex $v \in V$ choose arbitrarily a subset $F_{v} \subseteq E_{v}$ of cardinality $\delta$. Now randomly and independently for every vertex $v \in V$ choose one edge in $F_{v}$ - each with equal probability $\left(\right.$ i.e. $\left.\delta^{-1}\right)$ - and denote it by $e_{v}$. For every $v \in V$ denote the event:

- $A(v):\left|\left\{u \in N_{G}(v): e_{u} \neq u v\right\}\right|<10^{10}+1$. 
Suppose $v$ is a vertex of degree $d$; note that by the Chernoff Bound:

$$
\begin{aligned}
\mathbf{P} r(A(v)) & \leq \mathbf{P} r\left(\operatorname{BIN}\left(d, \frac{\delta-1}{\delta}\right)<10^{10}+1\right)<e^{-\frac{\left(\frac{\delta-1}{\delta} d-10^{10}-1\right)^{2}}{2 \frac{\delta-1}{\delta} d}} \\
& \leq e^{-\frac{\left(\frac{\delta-1}{\delta} d-\frac{\delta-1}{\delta} d\left(1-10^{-3}\right)\right)^{2}}{2 \frac{\delta-1}{\delta} d}}<e^{-2 \cdot 10^{-7} d},
\end{aligned}
$$

and set $x_{v}:=e^{-10^{-7} d}$ for such $v$. In order to apply the general version of the Local Lemma we define a dependency digraph $D$ by joining $A(v)$ with an arc to every $A(u)$ for which there exists $w \in V$ such that $u w, v w \in F_{w}$; note that there are at most $d \delta$ such events $A(u)$ for $v$. Then, since $e^{-x}<1-x+0,5 x^{2}$ for $x>0$ and $f(x):=e^{-10^{-8} x} x$ is decreasing for $x \geq 10^{8}$, we have:

$$
\begin{aligned}
x_{v} \prod_{A(u) \leftarrow A(v)}\left(1-x_{u}\right) & \geq e^{-10^{-7} d}\left(1-e^{-10^{-7} \delta}\right)^{\delta d} \\
& >e^{-10^{-7} d}\left(1-e^{-10^{-8} \delta}+0.5 e^{-2 \cdot 10^{-8} \delta}\right)^{\delta d} \\
& >e^{-10^{-7} d}\left(e^{-e^{-10^{-8} \delta}}\right)^{\delta d} \\
& >e^{-10^{-7} d}\left(e^{-e^{-10^{-8} 10^{10}} 10^{10}}\right)^{d} \\
& >e^{-2 \cdot 10^{-7} d} .
\end{aligned}
$$

By (12), (13) and Theorem 6 we thus conclude that there is a choice of edges $e_{u}, u \in V$, so that for every $v \in V$,

$$
\left|\left\{u \in N_{G}(v): e_{u} \neq u v\right\}\right| \geq 10^{10}+1 .
$$

It is then sufficient to set $S=\left\{e_{u}: u \in V\right\}$ to obtain $1 \leq d_{S}(v) \leq d(v)-10^{10}$ for each $v \in V$, as desired.

We are now ready to prove a lemma resembling one of the observations (i.e. Lemma 4.5) from [9] (used there as an ingredient in research concerning graph decompositions into a given finite number of locally irregular subgraphs). Lemma 16 above shall enable us to optimize the thesis of the aforementioned Lemma 17 below.

Lemma 17. Every graph $G=(V, E)$ without isolated edges can be decomposed into two graphs $H$ and $F$ such that: $H$ is either empty or has minimum degree $\delta(H) \geq 10^{10}$, and $F$ contains no isolated edges and has degeneracy less than $10^{10}+10^{8}$.

Proof. We shall first gradually remove some vertices from a given graph $G$. As long as there is still some vertex $v$ of degree less than $10^{10}+10^{8}$ in what is left of it, we remove $v$ from our contemporary graph. At the end of this process, we denote the leftover of $G$ by $H^{\prime}$ and let $F^{\prime}$ be the subgraph of $G$ induced by all its edges with at least one end outside $V\left(H^{\prime}\right)$. Note that $F^{\prime}$ has degeneracy less than $10^{10}+10^{8}$, while $\delta\left(H^{\prime}\right) \geq 10^{10}+10^{8}$ or 
$H^{\prime}$ is empty then. If $H^{\prime}$ is empty, the thesis holds. Otherwise, by Lemma 16, there exists $S \subseteq E\left(H^{\prime}\right)$ such that for every vertex $v \in V\left(H^{\prime}\right)$,

$$
1 \leq d_{S}(v) \leq d_{H^{\prime}}(v)-10^{10} .
$$

Note that for every isolated edge $u v$ of $F^{\prime}$, one of its ends must belong to $V\left(H^{\prime}\right)$ - we then arbitrarily choose one edge from $S$ incident with this end and add it to $F^{\prime}$ provided that no other edge adjacent to $u v$ was earlier added to $F^{\prime}$. After repeating this procedure for every such isolated edge we obtain a graph $F$ of $F^{\prime}$; note that the degeneracy of $F$ is still less than $10^{10}+10^{8}$ (as we may place the ends of the isolated edges of $F^{\prime}$ together with the vertices in $V(F) \backslash V\left(F^{\prime}\right)$ at the end of the ordering witnessing the degeneracy of $F$, since these vertices induce a forest in $F$ ). At the same time, by (14), the remaining subgraph of $G$, denoted by $H$ (formed from $H^{\prime}$ by removing edges from $S$ transferred to $F^{\prime}$ ), fulfills: $\delta(H) \geq 10^{10}$.

Theorem 18. Every graph $G$ without isolated edges can be decomposed into 24 subgraphs fulfilling the 1-2-3 Conjecture.

Proof. By Lemma 17, $G$ can be decomposed into a graph $H$ which is either empty or has minimum degree $\delta(H) \geq 10^{10}$ and a graph $F$ of degeneracy less than $10^{10}+10^{8}$ which contains no isolated edges. By Theorem 3, $H$ can be further decomposed into 3 locally irregular subgraphs (which obviously fulfill the 1-2-3 Conjecture). On the other hand, as $\chi(F) \leq 10^{10}+10^{8}<3^{21}$, by Lemma 10, $F$ can be decomposed into 21 graphs which are 3-colourable and contain no isolated edges, and thus fulfill the 1-2-3 Conjecture by Theorem 2 ,

\section{Concluding Remarks}

Note that by Theorems 8 and 15 we know that any $d$-regular graph without isolated edges can be decomposed into 2 subgraphs fulfilling the 1-2-3 Conjecture if only $d \notin$ $\{10,11,12,13,15,17\}$. The remaining cases apparently need a separate special treatment, but either way, by Corollary 12 every $d$-regular graph, $d \geq 2$, can be decomposed into (at most) 3 subgraphs complying with the 1-2-3 Conjecture. An even more challenging task was proposed in [7], whose authors suspect that something stronger should hold. Namely, they conjectured that in fact every graph $G$ without isolated edges and isolated triangles can be decomposed into 2 subgraphs fulfilling the 1-2-3 Conjecture with only weights 1 and 2 (i.e., admitting neighbour sum-distinguishing 2-edge-weightings). This interesting problem is independent of the 1-2-3 Conjecture itself, and is also partly related to the research from [9] - it is in particular known that this conjecture holds for bipartite graphs and subcubic graphs. See [7] for details and further observations concerning this new concept, and many other related problems and results.

\section{References}

[1] L. Addario-Berry, K. Dalal, C. McDiarmid, B.A. Reed, A. Thomason, Vertex-Colouring EdgeWeightings, Combinatorica 27(1) (2007) 1-12.

[2] L. Addario-Berry, K. Dalal, B.A. Reed, Degree Constrained Subgraphs, Discrete Appl. Math. 156(7) (2008) 1168-1174. 
[3] M. Aigner, E. Triesch, Irregular assignments of trees and forests, SIAM J. Discrete Math. 3(4) (1990) 439-449.

4] N. Alon, J.H. Spencer, The Probabilistic Method, 2nd edition, Wiley, New York, 2000.

[5] O. Baudon, J. Bensmail, J. Przybyło, M. Woźniak, On decomposing regular graphs into locally irregular subgraphs, European J. Combin. 49 (2015) 90-104.

[6] O. Baudon, J. Bensmail, É. Sopena, On the complexity of determining the irregular chromatic index of a graph, J. Discrete Algorithms 30 (2015) 113-127.

[7] O. Baudon, J. Bensmail, T. Davot, H Hocquard, J. Przybyło, M. Senhaji, É. Sopena, M. Woźniak, A general decomposition theory for the 1-2-3 Conjecture and locally irregular decompositions, submitted (https://hal.archives-ouvertes.fr/hal-01690222).

[8] J. Bensmail, A 1-2-3-4 result for the 1-2-3 Conjecture in 5-regular graphs, submitted (https://hal.archives-ouvertes.fr/hal-01509365/).

[9] J. Bensmail, M. Merker, C. Thomassen, Decomposing graphs into a constant number of locally irregular subgraphs, European J. Combin. 60 (2017) 124-134.

[10] G. Chartrand, P. Erdős, O.R. Oellermann, How to Define an Irregular Graph, College Math. J. 19(1) (1988) 36-42

[11] G. Chartrand, M.S. Jacobson, J. Lehel, O.R. Oellermann, S. Ruiz, F. Saba, Irregular networks, Congr. Numer. 64 (1988) 197-210.

[12] B. Cuckler, F. Lazebnik, Irregularity Strength of Dense Graphs, J. Graph Theory 58(4) (2008) 299-313.

[13] R.J. Faudree, M.S. Jacobson, J. Lehel, R. Schelp, Irregular networks, regular graphs and integer matrices with distinct row and column sums, Discrete Math. 76 (1989) 223-240.

[14] R.J. Faudree, J. Lehel, Bound on the irregularity strength of regular graphs, Colloq Math Soc Jańos Bolyai, 52, Combinatorics, Eger North Holland, Amsterdam, (1987), 247-256.

[15] A. Frieze, R.J. Gould, M. Karoński, F. Pfender, On Graph Irregularity Strength, J. Graph Theory 41(2) (2002) 120-137.

[16] S. Janson, T. Łuczak, A. Ruciński, Random Graphs, Wiley, New York, 2000.

[17] M. Kalkowski, A note on 1,2-Conjecture, in Ph.D. Thesis, 2009. Available at: https://repozytorium.amu.edu.pl/bitstream/10593/445/1 /Rozprawa\%20Doktorska\%202010\%20Maciej\%20Kalkowski.pdf

[18] M. Kalkowski, M. Karoński, F. Pfender, A new upper bound for the irregularity strength of graphs, SIAM J. Discrete Math. 25 (2011) 1319-1321.

[19] M. Kalkowski, M. Karoński, F. Pfender, Vertex-coloring edge-weightings: Towards the 1-2-3 conjecture, J. Combin. Theory Ser. B 100 (2010) 347-349.

[20] M. Karoński, T. Łuczak, A. Thomason, Edge weights and vertex colours, J. Combin. Theory Ser. B 91 (2004) 151-157.

[21] J. Lehel, Facts and quests on degree irregular assignments, Graph Theory, Combinatorics and Applications, Willey, New York, 1991, 765-782.

[22] B. Lužar, J. Przybyło, R. Soták, New bounds for locally irregular chromatic index of bipartite and subcubic graphs, submitted.

[23] P. Majerski, J. Przybyło, On the irregularity strength of dense graphs, SIAM J. Discrete Math. 28(1) (2014) 197-205.

[24] M. Molloy, B. Reed, Graph Colouring and the Probabilistic Method, Springer, Berlin, 2002.

[25] T. Nierhoff, A tight bound on the irregularity strength of graphs, SIAM J. Discrete Math. 13(3) (2000) 313-323.

[26] J. Przybyło, Irregularity strength of regular graphs, Electron. J. Combin. 15(1) (2008) $\sharp \mathrm{R} 82$.

[27] J. Przybyło, Linear bound on the irregularity strength and the total vertex irregularity strength of graphs, SIAM J. Discrete Math. 23(1) (2009) 511-516.

[28] J. Przybyło, On decomposing graphs of large minimum degree into locally irregular subgraphs, Electron. J. Combin. 23(2) (2016) $\sharp \mathrm{P} 2.31$

[29] J. Przybyło, M. Woźniak, On a 1,2 Conjecture, Discrete Math. Theor. Comput. Sci. 12(1) (2010) 101-108.

[30] C. Thomassen, Y. Wu, C.Q. Zhang, The 3-flow conjecture, factors modulo $k$, and the 1-2-3 conjecture, J. Combin. Theory Ser. B 121 (2016) 308-325.

[31] T. Wang, Q. Yu, On vertex-coloring 13-edge-weighting, Front. Math. China 3(4) (2008) 581-587. 